\title{
CAPACIDADE GERENCIAL DA ATENÇÃO PRIMÁRIA À SAÚDE PARA O CONTROLE DA TUBERCULOSE EM DIFERENTES REGIÕES DO BRASIL
}

\author{
Tereza Cristina Scatena Villa1, Maria Eugenia Firmino Brunello², Rubia Laine de Paula Andrade ${ }^{3}$, Nathalia \\ Halax Orfão ${ }^{4}$, Aline Aparecida Monro ${ }^{5}$, Jordana de Almeida Nogueira ${ }^{6}$, Reinaldo Antonio da Silva-Sobrinho ${ }^{7}$ \\ Erika Simone Galvão Pinto ${ }^{8}$, Silvia Helena de Figueiredo Vendramini ${ }^{9}$, Lucia Marina Scatena ${ }^{10}$, Maria Catarina \\ Salvador da Motta ${ }^{11}$, Antonio Ruffino Netto ${ }^{12}$
}

\footnotetext{
${ }^{1}$ Doutora em Enfermagem em Saúde Pública. Professora do Departamento de Enfermagem Materno-infantil e Saúde Pública da Escola de Enfermagem de Ribeirão Preto (EERP) da Universidade de São Paulo (USP). Ribeirão Preto, São Paulo, Brasil. E-mail: tite@eerp.usp.br

${ }^{2}$ Doutora em Enfermagem. Professora do Departamento de Enfermagem Materno-infantil e Saúde Pública da EERP/USP. Ribeirão Preto, São Paulo, Brasil. E-mail: mefb_usp@yahoo.com.br

${ }^{3}$ Doutora em Ciências. Especialista de laboratório do Departamento de Enfermagem Materno-infantil e Saúde Pública da EERP/ USP. Ribeirão Preto, São Paulo, Brasil. E-mail: rubia@eerp.usp.br

${ }^{4}$ Doutora em Ciências. Professora do Departamento de Enfermagem da Universidade Federal de Rondônia. Porto Velho, Rondônia, Brasil. E-mail: nathaliahalax@gmail.com

${ }^{5}$ Doutora em Enfermagem. Professora do Departamento de Enfermagem Materno-Infantil e Saúde Pública EERP/USP. Ribeirão Preto, São Paulo, Brasil. E-mail: amonroe@eerp.usp.br

${ }^{6}$ Doutora em Enfermagem. Professora do Departamento de Enfermagem Clínica da Universidade Federal da Paraíba. João Pessoa, Paraíba, Brasil. E-mail: jalnogueira31@gmail.com

${ }^{7}$ Pós-doutor em Ciências. Professor da Universidade Estadual do Oeste do Paraná. Foz do Iguaçu, Paraná, Brasil. E-mail: reisobrinho@yahoo.com.br

${ }^{8}$ Doutora em Enfermagem em Saúde Pública. Professora do Departamento de Enfermagem da Universidade Federal do Rio Grande do Norte. Natal, Rio Grande do Norte, Brasil. E-mail: erikasgp@gmail.com

${ }^{9}$ Doutora em Enfermagem em Saúde Pública. Professora de Enfermagem da Faculdade de Medicina de São José do Rio Preto. Ribeirão Preto, São Paulo, Brasil. E-mail: silviahve@gmail.com

${ }^{10}$ Doutora em Enfermagem em Saúde Pública. Professora do Departamento de Medicina Social da Universidade Federal do Triângulo Mineiro. Uberaba, Minas Gerais, Brasil. E-mail: 1mscatena@uol.com.br

${ }^{11}$ Doutora em Enfermagem em Saúde Pública. Professora da Escola de Enfermagem Anna Nery da Universidade Federal do Rio de Janeiro. Rio de Janeiro, Rio de Janeiro, Brasil. E-mail: ma.catarina@gmail.com

${ }^{12}$ Doutor em Ciências. Professor do Departamento de Medicina Social da Faculdade de Medicina de Ribeirão Preto da USP. Ribeirão Preto, São Paulo, Brasil. E-mail: aruffino@fmrp.usp.br
}

\section{RESUMO}

Objetivo: analisar a capacidade gerencial da atenção primária à saúde para o controle da tuberculose.

Método: pesquisa avaliativa, de delineamento transversal. População: profissionais de saúde da atenção primária à saúde que atuavam no controle da tuberculose. Coleta de dados: instrumento e questionário baseados na proposta de avaliação da capacidade institucional local, para o modelo de atenção às condições crônicas, adaptado para a atenção à tuberculose. Na análise dos dados, calculou-se o escore médio das respostas a cada questão do instrumento e realizou-se a análise de correspondência múltipla.

Resultados: a capacidade gerencial, na atenção primária à saúde, para o controle da tuberculose, apresentou resultados desfavoráveis para: agendamento no sistema de registro informatizado de consulta médica e exames de laboratório no tratamento de tuberculose, não utilização de comunicação formal e escrita para a referência dos casos de tuberculose. Além disso, o agente comunitário de saúde não se integra à equipe da unidade e não faz articulação com os recursos da comunidade e o apoio de especialista e a capacitação dos profissionais de saúde para o tratamento da tuberculose é limitado.

Conclusão: a capacidade organizacional dos serviços de atenção primária à saúde, nos municípios pesquisados, apresenta avaliação desfavorável para o acesso aos sistemas de monitoramento/vigilância, planejamento, capacitação e articulação com a comunidade para o controle da tuberculose, quesitos os quais influenciam a viabilização das políticas para mudanças no modelo de atenção à tuberculose como condição crônica.

DESCRITORES: Tuberculose. Avaliação de serviços de saúde. Atenção primária à saúde. Pesquisa operacional. Gestão em saúde. 


\title{
MANAGEMENT CAPACITY OF PRIMARY HEALTH CARE FOR TUBERCULOSIS CONTROL IN DIFFERENT REGIONS OF BRAZIL
}

\begin{abstract}
Objective: to analyze the management capacity of primary health care for tuberculosis control.

Method: cross-sectional study. Population: primary health care professional active in tuberculosis control. Data collection: instrument and questionnaire based on evaluation proposal of local institutional capacity for the chronic illness care model, adapted for tuberculosis care. In the data analysis, the mean score of answers to each instrument question was calculated and multiple correspondence analysis was applied.

Results: in primary health care, the management capacity for tuberculosis control presented unfavorable results for: scheduling the tuberculosis treatment medical consultation and laboratory tests in computerized registration system, non-use of formal and written communication for the referral of tuberculosis cases. In addition, the community health worker does not interact with the service team and does not articulate with the community resources. Expert support and health professional training for tuberculosis treatment are limited.

Conclusion: the organizational capacity of the primary care services in the investigated cities presents a negative assessment for the access to the monitoring/surveillance systems, planning, training and community articulation for tuberculosis controls, aspects that influence the feasibility of the policies for changes in the care model for tuberculosis as a chronic condition.
\end{abstract}

DESCRIPTORS: Tuberculosis. Health services research. Primary health care. Operations Research. Health Management.

\section{CAPACIDAD GERENCIAL DE LA ATENCIÓN PRIMARIA A LA SALUD PARA EL CONTROL DE LA TUBERCULOSIS EN DIFERENTES REGIONES DE BRASIL}

\begin{abstract}
RESUMEN
Objetivo: analizar la capacidad gerencial de la atención primaria a la salud para el control de la tuberculosis.

Método: investigación evaluativa, de delineamiento transversal. Población: profesionales de salud de la atención primaria a la salud que actuaban en el control de la tuberculosis. Recolección de datos: instrumento y cuestionario basados en la propuesta de evaluación de la capacidad institucional local, para el modelo de atención a las condiciones crónicas, adaptado para la atención a la tuberculosis. En el análisis de los datos, se calculó el puntaje medio de las respuestas a cada cuestión del instrumento y se realizó el análisis de correspondencia múltiple.

Resultados: la capacidad gerencial, en la atención primaria a la salud, para el control de la tuberculosis, presentó resultados desfavorables para: programación en el sistema de registro informatizado de consulta médica y exámenes de laboratorio en el tratamiento de tuberculosis, no utilización de comunicación formal y escrita para la intervención referencia de los casos de tuberculosis. Además, el agente comunitario de salud no se integra al equipo de la unidad y no hace articulación con los recursos de la comunidad y el apoyo de especialista y la capacitación de los profesionales de salud para el tratamiento de la tuberculosis es limitado.

Conclusión: la capacidad organizativa de los servicios de atención primaria a la salud, en los municipios encuestados, presenta una evaluación desfavorable para el acceso a los sistemas de monitoreo/vigilancia, planificación, capacitación y articulación con la comunidad para el control de la tuberculosis, que influyen en la viabilidad de las políticas para cambios en el modelo de atención a la tuberculosis como condición crónica
\end{abstract}

DESCRIPTORES: Tuberculosis. Investigación en servicios de salud. Atención primaria de salud. Investigación operativa. Gestión en salud.

\section{INTRODUÇÃO}

O presente artigo faz parte de uma série de pesquisas que vêm sendo desenvolvidas em três Regiões do Brasil, tendo-se como eixo a avaliação da organização e gestão dos serviços e sistemas de saúde para o controle da tuberculose (TB) na atenção primária à saúde (APS) dos municípios, considerando: acesso ao diagnóstico, ${ }^{1-2}$ tratamento de $\mathrm{TB}^{,-4}$ controle de comunicantes e cascata do cuidado. ${ }^{5}$ Para tanto, nos estudos são utilizados métodos e instrumentos de avaliação elaborados e adaptados para a atenção à $\mathrm{TB}$, na visão dos diversos atores envolvidos, a saber, doentes de $\mathrm{TB}$, profissionais de saúde e gestores.

Os contextos do estudo, no que se refere à organização do sistema de atenção à TB em siste- mas de saúde de três diferentes Regiões do Brasil, bem como os instrumentos de coleta de dados e referenciais teóricos, constituem desafios metodológicos e conceituais, uma vez que não há um único instrumento que consiga avaliar a multiplicidade das dimensões para a gestão do cuidado em saúde. ${ }^{6}$ A avaliação da execução de atividades específicas para a TB não pode ser dissociada da compreensão do contexto local, da estrutura e organização do sistema de saúde e dos aspectos que influenciam a viabilização das políticas vigentes.

Tais estudos são desenvolvidos em centros urbanos de três Regiões do Brasil que inovam ao contemplar, nos resultados, as diferenças, fragilidades/potencialidades e desafios para atenção à TB na APS, doença a qual demanda dos serviços de saúde e profissionais esforços que propiciem o cuidado mais 
amplo, multiprofissional e intersetorial., ${ }^{3,7}$

No Brasil, apesar da disponibilidade de maior oferta de ações de controle da TB na APS, a rede assistencial se encontra, na maioria das vezes, desarticulada, mal distribuída e mal equipada, ${ }^{8-10} \mathrm{e}$ os sistemas de saúde locais apresentam realidades complexas e heterogêneas nesse ponto de atenção, tais como o percentual de cobertura de unidades de atenção primária a saúde (UAPS), acesso ao sistema informatizado para assistência à TB na UAPS, o processo assistencial (oferta de ações médicas/ enfermagem, gerenciais e de acompanhamento dos casos de TB na UAPS).

Esses aspectos sinalizam a necessidade de estudos que abranjam indicadores e olhares voltados às peculiaridades relacionadas à estruturação da rede de saúde local até o processo de trabalho da equipe, na forma de acolher e prestar assistência aos doentes de TB.

Dificuldades identificadas em relação à incorporação das ações de controle da TB na APS têm mostrado a existência da estreita relação entre os fatores ligados ao insucesso do tratamento da TB e o modo como as ações de controle são desempenhadas nesse ponto de atenção. Entre os desafios para o controle da TB na APS, são identificados entraves relacionados ao fluxo de atendimento, à referência e contrarreferência, às debilidades de integração da equipe e dos níveis assistenciais e de ações e serviços, e à influência da falta de priorização das ações em TB na conformação da estrutura da rede para mudança de modelo de atenção e gestão. ${ }^{11-12}$

O gerenciamento e o controle da TB, como doença infecciosa, têm mais aspectos em comum com condições que envolvam cuidados em longo prazo, contínuos e coordenados a partir do nível da APS, e que ofertem ações que promovam adesão ao tratamento e mudança no estilo de vida. ${ }^{13} \mathrm{O}$ manejo da TB, portanto, ao ser encarado no escopo das condições crônicas, oferece evidências que subsidiam o planejamento de assistência que inclui aspectos individuais, sociais e culturais do doente, que almeja a corresponsabilização entre serviços de saúde, doente e família para o cuidado ${ }^{14-15}$ e, assim, requer o estabelecimento de uma rede de atenção integrada, que favoreça o acesso continuado dos usuários entre os diferentes pontos assistenciais e potencialize a articulação entre as instâncias gestoras, ${ }^{16}$ as quais envolvem a gestão da assistência, do serviço e do programa.

Considerando as dimensões para a avaliação da capacidade institucional no controle da TB, neste estudo aborda-se a dimensão organizacional do cuidado, definida como "aquela que se realiza nos serviços de saúde, marcada pela divisão técnica e social do trabalho, e evidencia novos elementos, como: o trabalho em equipe, as atividades de coordenação e comunicação, além da função gerencial propriamente dita. Nela, assume centralidade a organização do processo de trabalho, em particular, a definição de fluxos e regras de atendimento e a adoção de dispositivos compartilhados por todos os profissionais, tais como: as agendas, protocolos únicos, reuniões de equipe, planejamento, avaliação etc. Aqui, a gestão do cuidado depende da ação cooperativa de vários atores, a ser alcançada em territórios marcados, frequentemente, pelo dissenso, pela diferença, pelas disputas e pelas assimetrias de poder" ${ }^{6: 591}$

Dessa forma, o objetivo, neste estudo, foi analisar a capacidade gerencial da APS para o controle da TB, em três Regiões do Brasil.

\section{MÉTODO}

Pesquisa avaliativa, de delineamento transversal e abordagem quantitativa.

O estudo foi desenvolvido em municípios das Regiões Nordeste (Natal, RN; João Pessoa, PB), Sudeste (Uberaba, MG; São José do Rio Preto, SP; Rio de Janeiro, RJ - Área Programática (AP) 3.1, Complexo da Maré) e Sul (Foz do Iguaçu, PR) do Brasil. Tais municípios foram incluídos no estudo por serem prioritários para o controle da TB, bem como por terem descentralizado a realização das 
ações de controle da doença para a APS e apresentarem parceria entre equipes dos serviços de saúde e pessoal da academia.
Na tabela 1 apresentam-se a descrição dos dados populacionais e as informações epidemiológicas da TB nos locais de estudo:

Tabela 1 - Dados populacionais e informações epidemiológicas acerca da tuberculose de seis municípios de diferentes regiões do Brasil, 2013

\begin{tabular}{|c|c|c|c|c|c|c|}
\hline & $\begin{array}{l}\text { Foz do Iguaçu } \\
\text { (PR) }\end{array}$ & $\begin{array}{l}\text { Natal } \\
\text { (RN) }\end{array}$ & $\begin{array}{c}\text { João } \\
\text { Pessoa (PB) }\end{array}$ & $\begin{array}{l}\text { Uberaba } \\
\text { (MG) }\end{array}$ & $\begin{array}{c}\text { Complexo } \\
\text { da Maré (RJ) }\end{array}$ & $\begin{array}{l}\text { São José do } \\
\text { Rio Preto } \\
\text { (SP) }\end{array}$ \\
\hline População estimada & 255.718 & 869.954 & 769.607 & 315.360 & 129.770 & 434.039 \\
\hline Cobertura de ESF ${ }^{*}$ & $41,2 \%$ & $44,3 \%$ & $81,8 \%$ & $52,4 \%$ & $50,0 \%$ & $24,9 \%$ \\
\hline Número de casos de tuberculose & 126 & 311 & 506 & 54 & 170 & 136 \\
\hline \multicolumn{7}{|l|}{ Desfecho } \\
\hline Taxa de cura & $53,2 \%$ & $62,2 \%$ & $74,2 \%$ & $72,2 \%$ & $74,7 \%$ & $81,6 \%$ \\
\hline Taxa de abandono & $21,4 \%$ & $7,2 \%$ & $9,5 \%$ & $13,0 \%$ & $23,5 \%$ & $5,9 \%$ \\
\hline Taxa de óbito & $4,8 \%$ & $2,1 \%$ & $3,6 \%$ & $14,8 \%$ & $0,6 \%$ & $3,7 \%$ \\
\hline $\begin{array}{l}\text { Proporção de casos de tuber- } \\
\text { culose com acompanhamento } \\
\text { mensal na APS }\end{array}$ & $\begin{array}{l}\text { Sem informa- } \\
\text { ção }\end{array}$ & $100 \%$ & $19 \%$ & $12,0 \%$ & $100 \%$ & $65,9 \%$ \\
\hline $\begin{array}{l}\text { Proporção de casos de tubercu- } \\
\text { lose realizando TDO } \text { pela APS }^{\ddagger}\end{array}$ & $75,4 \%$ & $14,2 \%$ & $22 \%$ & $65,0 \%$ & $53,2 \%$ & $69,8 \%$ \\
\hline
\end{tabular}

"Estratégia Saúde da Família; ${ }^{\dagger}$ Atenção Primária à Saúde; ${ }^{\ddagger}$ Tratamento Diretamente Observado.

Fontes: IBGE, 2016 ${ }^{17}$; DAB, 2016 ${ }^{18}$; SINAN, 2016 ${ }^{19}$; TBWEB, $2016^{20}$

A população do estudo foi constituída de informantes-chave que compreenderam profissionais de saúde (enfermeiros, auxiliares e técnicos de enfermagem, Agentes Comunitários de Saúde (ACS) e médicos) dos serviços de APS, os quais atuavam no tratamento aos doentes de TB.

Os critérios de inclusão para a entrevista com profissionais de saúde foram: ser profissional de saúde atuante na APS no período de coleta de dados, de outubro de 2013 a fevereiro de 2014, e ter acompanhado, pelo menos uma vez, doentes de TB nos últimos seis meses anteriores à data da coleta.

Por meio do Cadastro Nacional de Estabelecimentos de Saúde, foi levantado o número de profissionais atuantes nos serviços de APS de cada município participante do estudo. Após esse levantamento, foi realizado contato com a coordenação do programa municipal de controle da TB, ou diretamente com as unidades de saúde, a fim de se identificar quantos e quais profissionais já haviam atendido e acompanhado doentes de TB.

Posteriormente, procedeu-se ao cálculo do tamanho amostral, considerando os seguintes parâmetros: erro amostral de 0,05; intervalo de confiança de $95 \%$ e $p$ (proporção populacional) de $50 \%$, com a seguinte equação:

$$
n_{0}=\frac{p(1-p) Z^{2}}{e^{2}}
$$

Por ter população finita, a amostra mínima de profissionais a ser entrevistada em cada município de estudo foi corrigida utilizando a equação abaixo.

$$
n=\frac{n_{0}}{1+\left(n_{0}-1 / N\right)}
$$

Dessa forma, foram entrevistados 618 profissionais, sendo 82 de João Pessoa, 100 de Natal, 173 do Rio de Janeiro, 70 de São José do Rio Preto, 100 de Uberaba e 93 de Foz do Iguaçu.

Para analisar a capacidade gerencial da APS, para o controle da TB, em três Regiões do Brasil, do cuidado em TB na APS, foram utilizadas diferentes fontes de coleta de dados, além de instrumentos e questionário distintos, sendo eles:

1) instrumento para a caracterização do sistema de atenção à TB dos municípios em relação à capacidade instalada, que continha informações tanto estruturais quanto do processo de trabalho das equipes para a assistência ao doente, tais como: número de Unidades Básicas de Saúde (UBS), Centros de Saúde (CS) e Unidade de Saúde da Família (USF); Tratamento Diretamente Observado (TDO), equipe que acompanha os casos de TB na UAPS, coordenação da assistência entre a equipe, coordenação do trabalho entre serviços de saúde, integração entre programas, serviços de saúde e profissionais, registros e sistema informatizado para assistência à TB, acesso às informações e processo assistencial (oferta de ações médicas, 
gerenciais e de acompanhamento dos casos de TB);

2) questionário estruturado baseado na proposta de MacCooll Institute for Health Care Innovation, Assessment of Chronic IllnessCare (ACIC) ${ }^{21}$, para a "avaliação da capacidade institucional local para desenvolver o modelo de atenção às condições crônicas", adaptado " para a avaliação das ações desenvolvidas para o controle da TB. Tal questionário é composto de questões que abordam a avaliação da atenção à TB nas seguintes dimensões: organização da atenção à TB; articulação com a comunidade; autocuidado apoiado; suporte à decisão; desenho do sistema de prestação de serviços; sistemas de informação clínica e integração dos componentes do modelo de atenção às pessoas com $\mathrm{TB}$, com as quais é possível obter as respostas em quatro níveis (A, B, C, D), sendo A o mais favorável, B e C, intermediários, e D, o mais desfavorável. As escalas de respostas foram representadas por valores que variavam de 0 a 11, sendo os valores de 0 a 2 alocados no nível D, 3 a 5, no C, 6 a 8, no B, e 9 a 11, no A.

Vale ressaltar que, para o presente estudo, apenas variáveis de algumas dimensões foram selecionadas, a fim de permitir a análise da gestão da atenção à TB conforme o conceito norteador da dimensão organizacional supracitado, com ênfase nos indicadores que pudessem avaliar os aspectos da capacidade gerencial da APS para a atenção à TB. Tais variáveis são expostas no quadro 1.

Na análise dos dados, calculou-se o escore médio das respostas a cada questão do instrumento, calculando-se também o desvio-padrão correspondente. Além disso, para identificar a existência de associação entre as variáveis ativas (Quadro 2) e a variável suplementar (municípios do estudo), procedeu-se a realização da análise de correspondência múltipla (ACM).

Na ACM, o maior percentual de variabilidade do conjunto de dados foi explicado pela combinação de variáveis com maior estabilidade no espaço multidimensional. As variáveis ativas detêm o papel principal na determinação dos resultados disponibilizados nos planos fatoriais. Feita a descrição do espaço no plano fatorial em função das associações entre as variáveis ativas, foi possível a inclusão da variável suplementar. A variável A1 (benefícios e incentivos) foi a única que apresentou menor valor próprio, sendo desconsiderada da análise por possuir menor estabilidade no espaço multidimensional.

\section{Quadro 1 - Variáveis ativas para avaliar os aspectos da capacidade gerencial da Atenção Primária a Saúde para a atenção à tuberculose}

\begin{tabular}{|c|c|}
\hline $\begin{array}{l}\text { dade ger- } \\
\text { la Atenção } \\
\text { a à Saúde } \\
\text { enção à }\end{array}$ & $\begin{array}{l}\text { G1 - Interesse do gerente da unidade de saúde em relação às mudanças na atenção à tuberculose } \\
\text { G2 - Metas pactuadas pela unidade de saúde para o controle da tuberculose na área de abrangência } \\
\text { G3 - Estratégias para melhoria da atenção à tuberculose } \\
\text { G4 - Estratégias para que a Atenção Primária à Saúde seja o local de tratamento da tuberculose } \\
\text { G5 - Participação do gerente da unidade de saúde para melhoria da atenção à tuberculose } \\
\text { G6 - Disponibilidade de manual de diretrizes para o controle da tuberculose na unidade de saúde } \\
\text { G7 - Realização de capacitação dos profissionais de saúde para a atenção à tuberculose } \\
\text { G8 - Monitoramento da situação da tuberculose na comunidade } \\
\text { G9 - Retorno de informações da vigilância/coordenação do Programa de Controle da Tuberculose } \\
\text { quanto ao desempenho da unidade } \\
\text { G10 - Planejamento da atenção à tuberculose inclui incentivos à unidade de saúde com base no } \\
\text { desempenho }\end{array}$ \\
\hline $\begin{array}{l}\text { a Atenção } \\
\text { à Saúde } \\
\text { רção à } \\
\text { ose }\end{array}$ & $\begin{array}{l}\text { A1 - Benefícios e incentivos aos portadores de tuberculose; } \\
\text { A2 - Parcerias com organizações da comunidade para o controle da tuberculose } \\
\text { A3 - Agente comunitário de saúde participa do tratamento da tuberculose e faz articulação com } \\
\text { equipe/comunidade } \\
\text { A4 - Participação de especialistas no apoio aos profissionais de saúde da unidade } \\
\text { A5 - Trabalho em equipe para o controle da tuberculose } \\
\text { A6 - Profissional de saúde da própria unidade que é referência para o controle da tuberculose } \\
\text { A7 - Sistema de agendamento de consultas para o tratamento da tuberculose } \\
\text { A8 - Continuidade do cuidado ao portador de tuberculose } \\
\text { A9 - Registro no prontuário clínico e informatização } \\
\text { A10 - Formulário de registro de informações dos portadores de tuberculose }\end{array}$ \\
\hline
\end{tabular}

Projeto Fapesp Regular "Avaliação da integração de serviços de saúde para o tratamento da tuberculose na Atenção Primária nas redes de atenção em regiões do Brasil". 
Finalmente, o padrão de interdependência entre as variáveis foi analisado por meio da análise de resíduos (diferença entre o observado e o esperado), em forma padronizada e ajustada, de tal modo que foram mantidos nos planos fatoriais somente variáveis com resíduos acima de 1,96, pois indicavam associação estatisticamente significante entre as variáveis. Dessa forma, foi possível criar um espaço fatorial para o conjunto de categorias das variáveis e interpretar suas associações. O nível de significância estatística adotado para as análises realizadas foi de $5 \%$.

O estudo foi aprovado pelo Comitê de Ética em Pesquisa da Escola de Enfermagem de Ribeirão Preto da Universidade de São Paulo, conforme protocolo CAAE 15671713.9.1001.53.93.

\section{RESULTADOS}

A cobertura da Unidade de Saúde da Família (USF), em relação aos dados populacionais no município, foi classificada em: acima de $80 \%$ (1); entre 40 e $55 \%$ (4) e abaixo de $25 \%$ (1), levando-se em consideração a maturidade e o tempo de implantação das ações de controle da TB na APS, em cinco municípios do estudo ocorreu há mais de cinco anos, e em um é recente.

Em relação à oferta de serviços, como a consulta médica (CM) de acompanhamento do tratamento de TB com agendamento regular, constatou-se ocorrência em três municípios (São José do Rio Preto SJRP; Rio de Janeiro - RJ; Uberaba - UB), e demanda espontânea não agendada, em três (João Pessoa - JP; Natal - NA; Foz do Iguaçu - FI). Em relação à oferta de TDO na UAPS, a cobertura foi classificada abaixo de $25 \%$ (2) e entre 50 e 75,4\% (4).

O sistema informatizado para assistência à TB na APS estava implantado para agendamento de $\mathrm{CM}$ e exames de laboratório em dois municípios, FI e SJRP. O RJ possuía sistema informatizado para exames laboratoriais à TB (GAL Prime). O sistema de referência para assistência à $\mathrm{TB}$ dos municípios não utilizava comunicação formal com registro e nem estavam implantadas as linhas-guia.

No lado positivo da dimensão 1 do plano fatorial (Figura 1), verificou-se capacidade gerencial favorável destinada à APS para o desenvolvimento de ações de controle da TB. Associados a tal resultado, foram encontrados os municípios do RJ e SJRP. No lado negativo da dimensão 1 desse plano fatorial, verificaram-se condições gerenciais desfavoráveis, de modo que, associado a essas, encontrou-se o município de UB. Ainda na Figura 1, os municípios de JP, NA e FI se aproximaram da origem da dimensão 1 e, por isso, representam o perfil médio dos itens avaliados, ou seja, foram avaliados de forma regular quanto à capacidade gerencial destinada à APS para o desenvolvimento de ações de controle da TB.

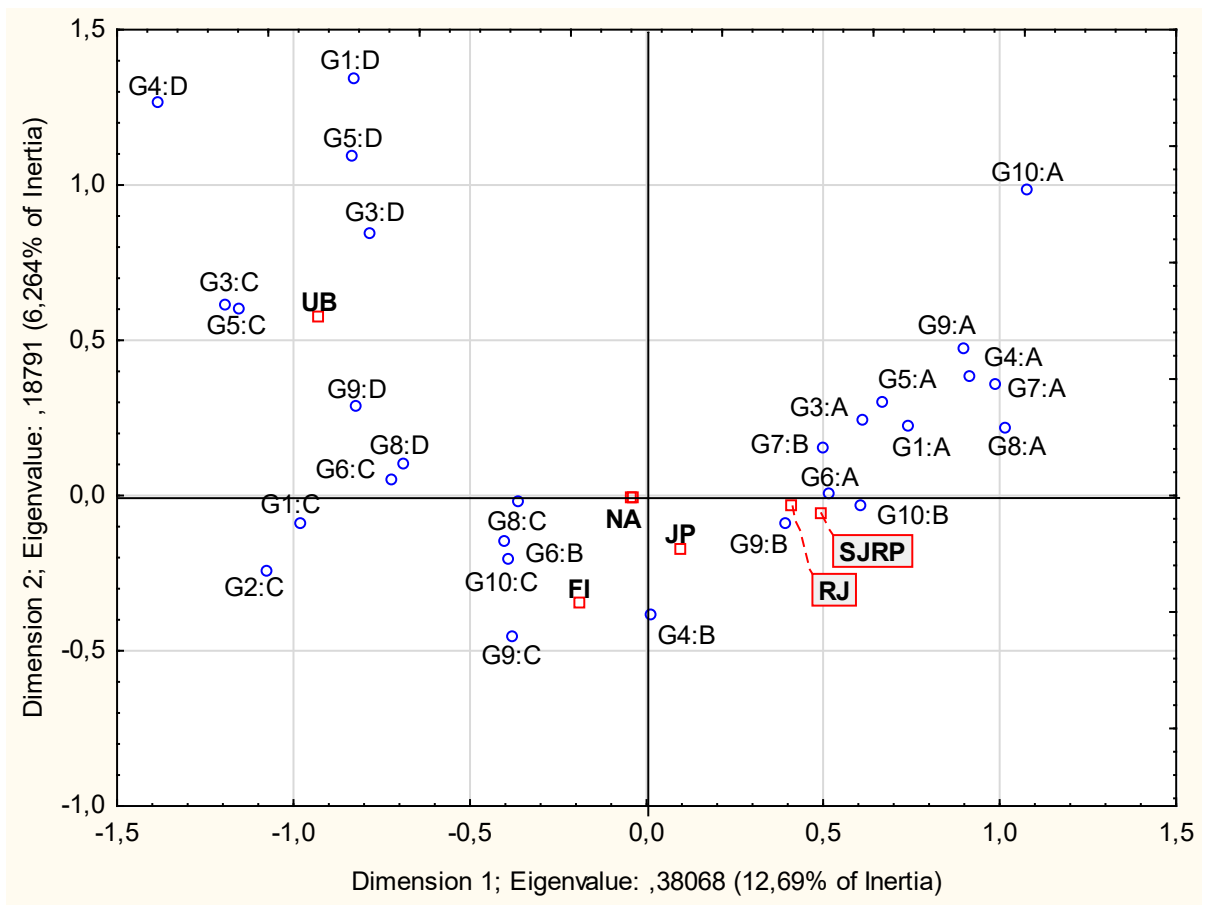

JP - João Pessoa; FI - Foz do Iguaçu; NA - Natal; RJ - Rio de Janeiro (Complexo da Maré); SJRP - São José do Rio Preto; UB - Uberaba; G1 Interesse do gerente da unidade de saúde em relação às mudanças na atenção à tuberculose; G2 - Metas pactuadas pela unidade de saúde para o controle da tuberculose na área de abrangência; G3 - Estratégias para melhoria da atenção à tuberculose; G4 - Estratégias para que 
a Atenção Primária à Saúde seja o local de tratamento da tuberculose; G5 - Participação do gerente da unidade de saúde para melhoria da atenção à TB; G6 - Disponibilidade de manual de diretrizes para o controle da tuberculose na unidade de saúde; G7 - Realização de capacitação dos profissionais de saúde para a atenção à tuberculose; G8 - Monitoramento da situação da tuberculose na comunidade; G9 - Retorno de informações da vigilância/coordenação do PCT quanto ao desempenho da unidade; G10 - Planejamento da atenção à tuberculose inclui incentivos à unidade de saúde com base no desempenho; A, B, C e D, sendo A capacidade mais favorável, B e C, intermediários, e D, mais desfavorável.

\section{Figura 1 - Capacidade gerencial destinada à Atenção Primária à Saúde para atenção à tuberculose em} municípios de três regiões do Brasil, 2013/2014

Esses resultados refletem os escores médios de cada item avaliado separadamente (Tabela 2). É possível, ainda, identificar que a variável avaliada desfavorável em todos os municípios do estudo diz respeito à realização de capacitação dos profissionais de saúde para a atenção à TB.

Tabela 2 - Análise descritiva da capacidade gerencial destinada à atenção primária à saúde para atenção à tuberculose em municípios de três Regiões do Brasil, 2013/2014

\begin{tabular}{ccccccc}
\hline & $\begin{array}{c}\text { João Pessoa } \\
\overline{\mathrm{x}} \pm \mathrm{dp}\end{array}$ & $\begin{array}{c}\text { Foz do Iguaçu } \\
\overline{\mathrm{x}} \pm \mathrm{d} p\end{array}$ & $\begin{array}{c}\text { Natal } \\
\overline{\mathrm{x}} \pm \mathrm{dp}\end{array}$ & $\begin{array}{c}\text { Rio de Janeiro } \\
\overline{\mathrm{x}} \pm \mathrm{dp}\end{array}$ & $\begin{array}{c}\text { São José do Rio Preto } \\
\text { Uberaba }\end{array}$ \\
\hline G1 & $8,02 \pm 2,45$ & $7,20 \pm 2,48$ & $7,37 \pm 3,12$ & $8,10 \pm 2,39$ & $8,73 \pm 2,63$ & $\overline{\mathrm{x}} \pm \mathrm{dp}$ \\
G2 & $8,43 \pm 2,60$ & $7,76 \pm 2,63$ & $7,65 \pm 3,04$ & $8,29 \pm 2,44$ & $8,76 \pm 2,99$ & $6,56 \pm 2,93$ \\
G3 & $7,84 \pm 2,83$ & $7,42 \pm 2,35$ & $8,11 \pm 2,82$ & $7,95 \pm 2,28$ & $9,29 \pm 2,30$ & $7,20 \pm 2,46$ \\
G4 & $7,76 \pm 2,30$ & $7,20 \pm 2,12$ & $7,81 \pm 1,92$ & $7,53 \pm 2,11$ & $8,27 \pm 1,78$ & $4,45 \pm 3,30$ \\
G5 & $8,37 \pm 2,16$ & $6,94 \pm 2,55$ & $7,16 \pm 2,67$ & $8,04 \pm 1,91$ & $7,47 \pm 2,52$ & $4,44 \pm 3,17$ \\
G6 & $7,34 \pm 3,13$ & $7,72 \pm 2,72$ & $8,13 \pm 3,01$ & $7,82 \pm 2,88$ & $9,61 \pm 1,91$ & $6,85 \pm 2,50$ \\
G7 & $4,40 \pm 3,35$ & $4,10 \pm 3,54$ & $4,64 \pm 3,30$ & $5,72 \pm 3,47$ & $5,00 \pm 3,04$ & $3,92 \pm 3,03$ \\
G8 & $5,91 \pm 2,35$ & $5,60 \pm 2,46$ & $5,13 \pm 2,77$ & $6,79 \pm 2,84$ & $6,30 \pm 2,53$ & $5,29 \pm 1,84$ \\
G9 & $5,83 \pm 3,22$ & $4,71 \pm 3,17$ & $5,17 \pm 3,16$ & $6,47 \pm 3,08$ & $6,57 \pm 2,56$ & $3,98 \pm 2,67$ \\
G10 & $5,67 \pm 1,74$ & $5,33 \pm 1,97$ & $5,11 \pm 2,05$ & $6,54 \pm 2,13$ & $6,30 \pm 1,88$ & $4,62 \pm 1,66$ \\
& $6,96 \pm 2,96$ & $6,40 \pm 2,91$ & $6,63 \pm 3,12$ & $7,32 \pm 2,72$ & $7,63 \pm 2,84$ & $5,20 \pm 2,99$ \\
\hline
\end{tabular}

G1 - Interesse do gerente da unidade de saúde em relação às mudanças na atenção à tuberculose; G2 - Metas pactuadas pela unidade de saúde para o controle da tuberculose na área de abrangência; G3 - Estratégias para melhoria da atenção à tuberculose; G4 - Estratégias para que a Atenção Primária à Saúde seja o local de tratamento da tuberculose; G5 - Participação do gerente da unidade de saúde para melhoria da atenção à tuberculose; G6 - Disponibilidade de manual de diretrizes para o controle da tuberculose na unidade de saúde; G7 - Realização de capacitação dos profissionais de saúde para a atenção à tuberculose; G8 - Monitoramento da situação da tuberculose na comunidade; G9 - Retorno de informações da vigilância/coordenação do PCT quanto ao desempenho da unidade; G10 - Planejamento da atenção à tuberculose inclui incentivos à unidade de saúde com base no desempenho.

Tanto na figura 2 quanto na tabela 3, a capacidade para a assistência à TB nos municípios apresentou variabilidade de respostas considerável, uma vez que a aproximação das categorias de resposta não seguiu distribuição lógica.

As variáveis mais bem avaliadas tiveram maior proximidade com o município do Rio de Janeiro, São José do Rio Preto teve avaliação intermediária, e os demais municípios ficaram dispersos entre respostas intermediárias e ruins.

A variável "benefícios e incentivos aos porta- dores de tuberculose" recebeu melhor avaliação nos municípios de João Pessoa e Natal.

Foi avaliada de forma desfavorável em todos os municípios a variável "parcerias com organizações da comunidade para o controle da tuberculose".

A variável "participação de especialistas no apoio aos profissionais de saúde da unidade" recebeu avaliação entre intermediária e desfavorável nos municípios do estudo, exceto em São José do Rio Preto, onde foi mais bem avaliada. 


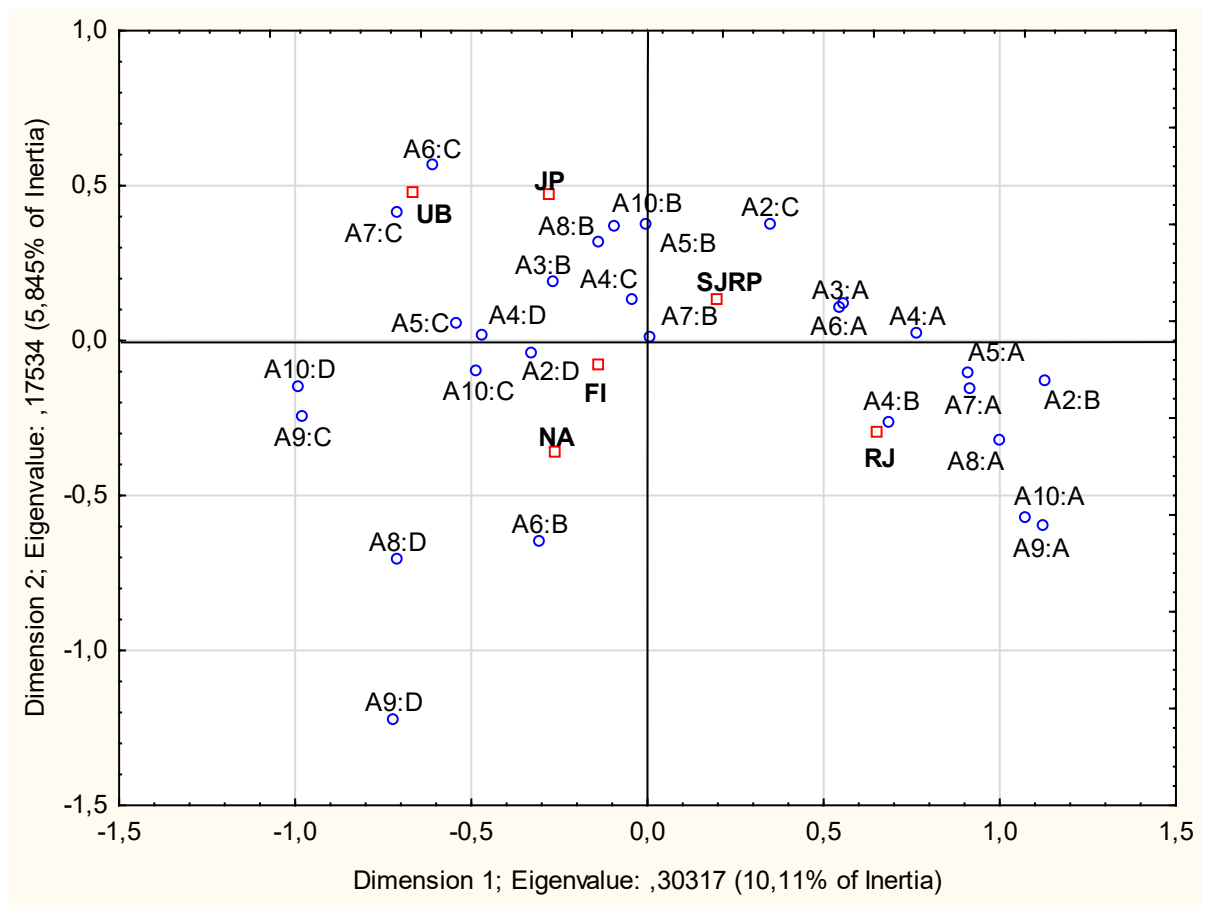

JP - João Pessoa; FI - Foz do Iguaçu; NA - Natal; RJ - Rio de Janeiro (Complexo da Maré); SJRP - São José do Rio Preto; UB - Uberaba; A1 - Benefícios e incentivos aos portadores de Tuberculose; A2 Parcerias com organizações da comunidade para o controle da tuberculose; A3 - Agente comunitário de saúde participa do tratamento da tuberculose e faz articulação com equipe/comunidade; A4 Participação de especialistas no apoio aos profissionais de saúde da unidade; A5 - Trabalho em equipe para o controle da tuberculose; A6 - Profissional de saúde da própria unidade que é referência para o controle da tuberculose; A7 - Sistema de agendamento de consultas para o tratamento da tuberculose; A8 - Continuidade do cuidado ao portador de tuberculose; A9 - Registro no prontuário clínico e informatização; A10 - Formulário de registro de informações dos portadores de tuberculose; A, B, C e D, sendo A capacidade mais favorável, B e C, intermediários, e D, mais desfavorável

Figura 2 - Capacidade da Atenção Primária à Saúde para assistência à tuberculose em municípios de três regiões do Brasil, 2013/2014

Tabela 3 - Análise descritiva da capacidade instalada da atenção primária à saúde para assistência aos casos de tuberculose em municípios de três regiões do Brasil, 2013/2014

\begin{tabular}{ccccccc}
\hline & $\begin{array}{c}\text { João Pessoa } \\
\overline{\mathrm{x}} \pm \mathrm{dp}\end{array}$ & $\begin{array}{c}\text { Foz do Iguaçu } \\
\overline{\mathrm{x}} \pm \mathrm{dp}\end{array}$ & $\begin{array}{c}\text { Natal } \\
\overline{\mathrm{x}} \pm \mathrm{dp}\end{array}$ & $\begin{array}{c}\text { Rio de Janeiro } \\
\overline{\mathrm{x}} \pm \mathrm{dp}\end{array}$ & $\begin{array}{c}\text { São José do Rio Preto } \\
\overline{\mathrm{x}} \pm \mathrm{dp}\end{array}$ & $\begin{array}{c}\text { Uberaba } \\
\overline{\mathrm{x}} \pm \mathrm{dp}\end{array}$ \\
\hline $\mathrm{A} 1$ & $7,46 \pm 2,97$ & $0,99 \pm 2,97$ & $8,44 \pm 2,29$ & $2,49 \pm 3,54$ & $3,40 \pm 3,72$ & $0,25 \pm 1,36$ \\
$\mathrm{~A} 2$ & $1,98 \pm 2,95$ & $1,08 \pm 1,91$ & $1,25 \pm 2,69$ & $3,25 \pm 3,30$ & $2,67 \pm 3,30$ & $0,91 \pm 2,00$ \\
A3 & $9,11 \pm 1,85$ & $7,47 \pm 2,04$ & $7,67 \pm 3,73$ & $8,61 \pm 1,99$ & $7,87 \pm 3,55$ & $7,74 \pm 1,43$ \\
A4 & $2,70 \pm 2,40$ & $5,19 \pm 2,96$ & $2,67 \pm 2,20$ & $4,97 \pm 3,13$ & $7,99 \pm 3,17$ & $3,29 \pm 2,62$ \\
A5 & $7,60 \pm 2,32$ & $5,41 \pm 2,52$ & $6,36 \pm 3,21$ & $7,38 \pm 2,97$ & $6,83 \pm 1,86$ & $6,47 \pm 2,32$ \\
A6 & $7,29 \pm 2,82$ & $7,27 \pm 2,84$ & $8,03 \pm 3,04$ & $8,16 \pm 2,78$ & $8,07 \pm 2,42$ & $5,89 \pm 2,93$ \\
A7 & $5,98 \pm 2,25$ & $6,81 \pm 2,58$ & $7,13 \pm 2,20$ & $7,85 \pm 2,32$ & $7,89 \pm 2,26$ & $5,76 \pm 2,44$ \\
A8 & $5,76 \pm 3,15$ & $7,19 \pm 2,98$ & $5,37 \pm 3,32$ & $7,60 \pm 2,81$ & $7,30 \pm 1,90$ & $6,66 \pm 2,04$
\end{tabular}


A9

A10
$7,29 \pm 1,46$

6,32 $\pm 1,91$

$6,15 \pm 3,24$
$7,45 \pm 1,69$

$6,35 \pm 2,93$

$5,52 \pm 3,45$
$7,32 \pm 1,63$

$6,68 \pm 2,08$

$6,09 \pm 3,51$
$8,02 \pm 2,15$

$8,19 \pm 2,43$

$6,65 \pm 3,50$
$7,46 \pm 1,59$

$6,59 \pm 2,03$

$6,61 \pm 3,25$
$5,67 \pm 2,06$

$5,91 \pm 2,43$

$4,85 \pm 3,25$

A1 - Benefícios e incentivos aos portadores de tuberculose; A2 - Parcerias com organizações da comunidade para o controle da tuberculose; A3 - Agente comunitário de saúde participa do tratamento da tuberculose e faz articulação com equipe/comunidade; A4 - Participação de especialistas no apoio aos profissionais de saúde da unidade; A5 - Trabalho em equipe para o controle da tuberculose; A6 - Profissional de saúde da própria unidade que é referência para o controle da tuberculose; A7 - Sistema de agendamento de consultas para o tratamento da tuberculose; A8 - Continuidade do cuidado ao portador de tuberculose; A9 - Registro no prontuário clínico e informatização; A10 Formulário de registro de informações dos portadores de tuberculose.

\section{DISCUSSÃO}

As experiências de descentralização das ações de TB para a APS demonstraram que os municípios se encontram em diferentes estágios e, também, que esse processo tem sido determinado pela especificidade local e por avanços no processo de gestão, sendo que cada município apresenta uma forma de organização de atenção à TB. A dificuldade de oferecer ações de controle da TB na APS evidenciada neste estudo, mesmo em municípios com média ou alta cobertura de USF, é uma realidade, pela baixa proporção de casos de TB acompanhados e de TDO realizado nesse ponto de atenção.

Em estudo em JP, município com mais de $80 \%$ de cobertura de USF, identificou-se que os profissionais da APS estimulam o doente a realizar o tratamento em ambulatório de referência para TB, muitas vezes por desconhecerem que a doença é uma das prioridades da APS, ignorando as políticas e dados epidemiológicos que a envolvem. ${ }^{22}$

Verifica-se que os profissionais da UAPS referem ter acesso aos manuais e diretrizes para atenção à $\mathrm{TB}$, no entanto, observa-se descompasso entre a transferência de responsabilidades das ações de controle da TB para as UAPS e a capacidade de resposta das equipes para a gestão do cuidado, em alguns municípios do estudo. Ademais, esses apresentam percentuais de cura e abandono abaixo das metas estabelecidas pelo Programa Nacional de Controle da Tuberculose.

Em estudos realizados no Nordeste do país, abordou-se a forma como os serviços de atenção básica são implantados, sem o suprimento necessário de recursos que garantam seu pleno funcionamento, e que levam à ineficiência na qualidade dos serviços. ${ }^{23}$

Os indicadores mostram que os gerentes das UAPS ainda apresentam dificuldades em pactuar ações voltadas para o controle da TB por meio de planejamento e com registro sistemático, sendo que as metas pactuadas não estão disponíveis para a equipe local.

Verifica-se ainda que, embora a APS tenha contribuído para ampliar o acesso da população aos serviços de saúde, o planejamento e o monitoramento das atividades desenvolvidas por esse ponto de atenção ainda são problemáticos. ${ }^{14,23-24}$

Os nós críticos da capacidade gerencial perpassam por fatores ligados tanto à expansão dos serviços em municípios cada vez mais populosos quanto ao financiamento, o qual afeta a disponibilidade de recursos humanos, ${ }^{23}$ especialmente médicos na Região Nordeste, para o acompanhamento dos doentes de TB, fazendo com que os profissionais da equipe de enfermagem assumam a maioria das ações de combate à doença. ${ }^{10}$

No que se refere à avaliação básica e limitada da oferta de benefícios e incentivos, em alguns municípios, em outro estudo realizado em um município do Nordeste brasileiro, mostrou-se que tal fragilidade pode levar à descontinuidade e ao abandono do tratamento, inviabilizando o cumprimento de metas e alcance da cura. ${ }^{25}$

A avaliação dos profissionais foi crítica em relação à criação de parcerias com organizações da comunidade, o que se verificou em outras pesquisas. ${ }^{26-28}$ Embora a participação do Agente Comunitário de Saúde nas atividades ligadas ao tratamento da TB tenha sido considerada razoável neste estudo, o mesmo não se integra à equipe da UAPS e não faz articulação com os recursos da comunidade. ${ }^{10}$

Em alguns municípios, verifica-se que os profissionais de saúde não recebem apoio de especialista e a capacitação para o tratamento da TB é deficiente. Dessa forma, há que se refletir sobre divergências e contradições quanto à efetividade da descentralização e à capacidade assistencial. Em alguns estudos, são apontados cenários favoráveis em relação ao alcance de indicadores de desfecho de tratamento, mesmo sem a incorporação de todos os compromissos e atividades. ${ }^{29-30}$ Em outros, identificou-se queda nas taxas de adesão e cura ${ }^{31}$ como também dificuldades de acesso ao tratamento e ações vinculadas ao mesmo, ${ }^{32-33}$ além de baixa integração entre ações e serviços de saúde no planejamento da assistência ao doente de TB. ${ }^{34}$

Os resultados ora mostrados apontam o baixo 
protagonismo da APS no desenvolvimento de ações para o controle da TB, sendo reflexo da falta de compromisso político que perpassa o nível gerencial, bem como da não responsabilização em relação às ações no âmbito assistencial. Com isso, para a atenção à TB na APS, é fundamental uma estrutura mínima nos serviços de saúde, com preparação das equipes locais e supervisão e retaguarda contínua da coordenação do PCT, para que as atividades sejam efetivas e respondam à realidade e necessidade local..$^{35}$

Neste estudo, verifica-se que a questão da atenção voltada e focalizada na comunidade aparece como a lacuna mais importante a ser vencida no direcionamento das ações de controle da TB. Tal deficiência também foi identificada em outros estudos nos quais se analisou o desempenho dos serviços de saúde para a atenção à TB em municípios do Estado de São Paulo. ${ }^{36-37}$

Verifica-se que é imprescindível a realização de reuniões regulares com vistas a planejar e coordenar o trabalho em equipe e a assistência de modo que se tenha como foco o controle da doença, ainda que existam ou não profissionais de saúde de referência para tanto na unidade. Portanto, a abordagem tradicional para as necessidades globais precisa ser reavaliada com maior ênfase em colaboração multidisciplinar e estratégias integradas. ${ }^{35}$

O sistema de informação clínica para a assistência à TB requer dos profissionais a utilização de diversos instrumentos que precisam ser preenchidos de forma manual, o que dificulta o compartilhamento e uso efetivo das informações. Cabe destacar ainda que os sistemas de informação da TB (Sinan e TB-WEB) não estão disponíveis ao acesso de profissionais da APS, necessitando que a informação também seja repassada por escrito. ${ }^{38}$

A atenção à TB avaliada por meio de fontes e dados epidemiológicos dos municípios em estudo explicita resultados desfavoráveis de cura, abandono do tratamento e óbito.

Como limitação neste estudo identifica-se o desafio, de ordem metodológica e conceitual, que diz respeito à realização do estudo com profissionais de saúde apenas, os quais podem fazer avaliação mais positiva do evento estudado em relação ao que ocorre na realidade da assistência prestada.

\section{CONCLUSÃO}

Desafios estão presentes na capacidade gerencial e assistencial da APS para o controle da TB, uma vez que os serviços desse nível de atenção não apresentaram resultados de tratamento favoráveis nos municípios, com altas taxas de abandono e óbito.

É possível identificar que há obstáculos para desenvolver a capacidade organizacional dos serviços de APS para o controle da TB nos municípios pesquisados, no que diz respeito aos aspectos que influenciam a viabilização das políticas vigentes para mudanças no modelo de atenção. Esse apresenta restrições de transferência de tecnologias de gestão, como o acesso aos sistemas de registro e informatização para o acompanhamento terapêutico, monitoramento/vigilância e planejamento de ações e capacitação dos profissionais.

Outra barreira refere-se à divisão social e técnica do trabalho em saúde e na enfermagem com maior ou menor presença do ACS na equipe, baixa disponibilidade de profissionais para o acompanhamento dos doentes de TB, como médicos em municípios do Nordeste e Sul, e pela incipiente retaguarda de especialistas e articulações com as organizações da comunidade no controle da doença. Tal fato acarreta assistência inadequada que influencia a viabilização das políticas para mudanças no modelo de atenção à TB como condição crônica e que varia em qualidade entre as Regiões do Brasil.

Aponta-se, ainda, a necessidade de acesso a sistemas informatizados de TB e integrados que estejam disponíveis à equipe de saúde para a gestão da assistência do doente de TB na APS, visto que os indicadores apontados no presente estudo, bem como as taxas desfavoráveis nos desfechos notificados, mostram-se preocupantes.

\section{AGRADECIMENTOS}

DECIT/CNPq - processo 404073/2012-3: “Tuberculose: análise dos pontos de estrangulamento da atenção para o controle da doença em municípios das Regiões Sul, Sudeste e Nordeste do Brasil" e FAPESP Regular - 2013/22512-3: “Avaliação da integração de serviços de saúde para o tratamento da tuberculose na atenção primária nas redes de atenção em Regiões do Brasil".

\section{REFERÊNCIAS}

1. Villa TCS, Ponce MAS, Wysocki AD, Andrade RLP, Arakawa T, Scatolin BE, et al. Early diagnosis of tuberculosis in the health services in different regions of Brazil. Rev Latino-am Enfermagem [Internet] 2013 Jan/Feb [cited 2017 Mar 13]; 21(spe):190-8. Available from: http:/ / www.scielo.br/scielo.php?script=sci_ar ttext\&pid=S0104-11692013000700024.

2. Silva Sobrinho RA, Ponce MAS, Andrade RLP, Beraldo AA, Pinto ESG, Scatena LM, et al. Effectiveness in 
the diagnosis of tuberculosis in Foz do Iguaçu, the triple-border area of Brazil, Paraguay and Argentina. Rev Esc Enferm USP [Internet]. 2013 Dec [cited 2017 Mar 13]; 47(6):1371-8. Available from: http:/ /www. scielo.br/scielo.php?script=sci_arttext\&pid=S008062342013000601373\&lng=en\&nrm=iso\&tlng=en.

3. Silva DM, Farias HBG, Villa TCS, Sá LD, Brunello MEF, Nogueira JA. Care production for tuberculosis cases: analysis according to the elements of the Chronic Care Model. Rev Esc Enferm USP [Internet]. 2016 Mar/ Apr [cited 2017 Mar 13]; 50(2):239-46. Available from: http:/ / www.scielo.br/scielo.php?script=sci_arttext\& pid=S0080-62342016000200239.

4. Villa TCS, Andrade RLP, Arakawa T, Magnabosco GT, Beraldo AA, Monroe AA, et al. Satisfação do usuário com os serviços de atenção à tuberculose em Ribeirão Preto, 2008. Cad Saúde Colet [Internet]. 2012 [cited 2017 Mar 13]; 20(2):234-43. Available from: http:// iesc.ufrj.br/cadernos/images/csc/2012_2/artigos/ csc_v20n2_234-243.pdf

5. Wysocki AD, Villa TCS, Arakawa T, Brunello MEF, Vendramini SHF, Monroe AA, et al. Latent tuberculosis infection diagnostic and treatment cascade among contacts in Primary Health Care in a city of Sao Paulo State, Brazil: cross-sectional study. PlosOne [Internet]. 2016 Jun [cited 2017 Mar 13]; 11(6):e0155348. Available from: http:/ /journals. plos.org/plosone/article?id=10.1371/journal. pone. 0155348

6. Cecilio LCO. Apontamentos teórico-conceituais sobre processos avaliativos considerando as múltiplas dimensões da gestão do cuidado em saúde. Interface (Botucatu) [Internet]. 2011 Apr/Jun [cited 2017 Feb 22]; 15(37):589-99. Available from: http://dx.doi. org/10.1590/S1414-32832011000200021.

7. Scatena LM, Wysocki AD, Beraldo AA, Magnabosco GT, Brunello MEF, Ruffino Netto A, et al. Validity and reliability of a health care service evaluation instrument for tuberculosis. Rev Saúde Pública [Internet]. 2015 Feb [cited 2017 Mar 13]; 49:7. Available from: http:/ / www.scielo.br/scielo.php?script=sci_ar ttext\&pid=S0034-89102015000100213.

8. Oliveira CBB, Assolini FEP, Protti ST, Souza KMJ, Monroe AA, Palha PF, et al. Management in Primary Health Care: discourses about the search for respiratory symptomatics of tuberculosis. Texto Contexto Enferm [Internet]. 2016 Oct [cited 2017 Mar 13]; 25(3):1-8. Available from: http:/ / www.scielo.br/scielo.php?script=sci_arttext\&pid =S0104-07072016000300315.

9. Arakawa T; Magnabosco GT, Lopes LM, Arnaez MAA, Gavín MAO, Gallardo MDPS, et al. Evaluation of the performance of Tuberculosis Control Programs in Brazil and Spain: an integrative review of the literature. Ciênc Saúde Coletiva [Internet]. 2015 Dec [cited 2017 Mar 13]; 20(12):3877-89. Available from: http:/ / www.scielo.br/scielo.php?script=sci_ arttext\&pid=S1413-81232015001203877\&lng=en\&nr $\mathrm{m}=$ iso\&tlng=en.

10. Silva DM, Nogueira JÁ, Sá LD, Wysocki AD, Scatena LM, Villa TCS. Performance evaluation of primary care services for the treatment of tuberculosis. Rev Esc Enferm USP [Internet]. 2014 Dec [cited 2017 Mar 13]; 48(6):1044-53. Available from: http:// www.scielo.br/scielo.php?script=sci_arttext\&pid $=$ S0080-62342014000601044

11. Brunello MEF, Andrade RLP, Monroe AA, Arakawa T, Magnabosco GT, Orfão NH, et al. Tuberculosis diagnostic pathway in a municipality in southeastern Brazil. Int J Tuberc Lung Dis [Internet]. 2013 Oct [cited 2017 Mar 13]; 17(Suppl 1):41-7. Available from: http://www.ingentaconnect.com/contentone/ iuatld/ijtld/2013/00000017/A00110s1/art00010.

12. Assis EG, Beraldo AA, Monroe AA, Scatena LM, Cardozo Gonzales RI, Palha PF, et al. The coordination of care for tuberculosis control. Rev Esc Enferm USP [Internet]. 2012 Feb [cited 2017 Mar 13]; 46(1):111-8. Available from: http:/ / www. scielo.br/scielo.php?script=sci_arttext\&pid=S008062342012000100015\&lng=en\&nrm=iso\&tlng=en

13. Escorel S, Giovanella L, Mendonça MHM, Senna MCM. O Programa de Saúde da Família e a construção de um novo modelo para a atenção básica no Brasil. Rev Panam Salud Publica [Internet]. 2007 [cited 2017 Mar 14]; 21 (2/3): 164-76. Available from: http:// www.scielosp.org/pdf/rpsp/v21n2-3/11.pdf.

14. Barrêto AJR, Sá LD, Nogueira JA, Palha PF, Pinheiro PGOD, Farias NMP et al. Organização dos serviços de saúde e a gestão do cuidado à tuberculose. Ciênc saúde coletiva [Internet]. 2012 Jul [cited 2017 Mar 13]; 17(7):1875-84. Available from: http://www.scielo.br/scielo.php?script=sci_ arttext\&pid=S1413-81232012000700027

15. Cunha NV, Cavalcanti MLT, Costa AJL. Diagnóstico situacional da descentralização do controle da tuberculose para a Estratégia Saúde da Família em Jardim Catarina, São Gonçalo, RJ, 2010. Cad Saúde Coletiva [Internet]. 2012 [cited 2017 Mar 13]; 20(2):177-87. Available from: http:/ / www.cadernos. iesc.ufrj.br/cadernos/images/csc/2012_2/artigos/ csc_v20n2_177-187.pdf.

16. Almeida PF, Giovanella L, Mendonça MHM, Escorel S. Desafios à coordenação dos cuidados em saúde: estratégias de integração entre níveis assistenciais em grandes centros urbanos. Cad Saúde Pública [Internet]. 2010 Feb [cited 2017 Feb 23]; 26(2):286-98. Available from: http://dx.doi.org/10.1590/S0102311X2010000200008.

17. Instituto Brasileiro de Geografia e Estatística. IBGE cidades [internet]. Brasília (DF): 2016 [cited 2016 Nov 26]. Available from: https://cidades.ibge.gov.br/ brasil/panorama

18. DepartamentodeAtençãoBásica.InformaçãoeGestãoda Atenção Básica. Cobertura da Atenção Básica [internet]. Brasília (DF): 2016 [cited 2016 Ago 15]. Available 
from: https:/ / egestorab.saude.gov.br/paginas/ acessoPublico/relatorios/relHistoricoCoberturaAB. xhtml;jsessionid=UKM2sWYlg9daAiyxXOyd9n1f

19. Departamento de Processamento de Dados do Sistema Único de Saúde. Sistema de Informação de Agravos de Notificação SINAN. Brasília (DF): 2016 [cited 2016 Out 02]. Available from: http://www2.datasus.gov. $\mathrm{br} /$ DATASUS $/$ index.php?area $=0203 \& \mathrm{id}=3100940$ 7\&VObj=http://tabnet.datasus.gov.br/cgi/tabcgi. exe?sinannet/cnv/tuberc

20. Governo do Estado de São Paulo. Centro de Vigilância Epidemiológica Prof. Alexandre Vranjac. Sistema de controle de pacientes com tuberculose TB-WEB. São Paulo (SP): 2016 [cited 2016 Out 27]. Available from: http://www.cvetb.saude.sp.gov.br/tbweb/index.jsp (acesso restrito).

21. Moysés ST, Silveira Filho AD, Moysés SJ. Laboratório de inovações no cuidado das condições crônicas na APS: a implantação do Modelo de Atenção às Condições Crônicas na UBS Alvorada em Curitiba, Paraná. Brasília (DF): Organização Pan-Americana da Saúde, Conselho Nacional de Secretários de Saúde; 2012.

22. Pinheiro PGOD, Sá LD, Palha PF, Barreto AJR, Rodrigues DCS, Romera AA. Discursividade de apoiadores matriciais sobre o controle da tuberculose e o modelo de gestão municipal. Rev Eletr Enf [Internet]. 2014 Jul/Sep [cited 2017 Mar 14]; 16(3): 502-10, 2014. Available from: https://www.fen.ufg.br/revista/ v16/n3/pdf/v16n3a02.pdf.

23. Pinafo E, Carvalho BG, Nunes EFPA. Descentralização da gestão: caminho percorrido, nós críticos e perspectivas. Ciênc Saúde Coletiva [Internet]. 2016 May [cited 2017 Feb 22]; 21(5):1511-24. Available from: http://dx.doi.org/10.1590/141381232015215.18942015.

24. Turci MA, Lima-Costa MF, Macinko J. Influência de fatores estruturais e organizacionais no desempenho da atenção primária à saúde em Belo Horizonte, Minas Gerais, Brasil, na avaliação de gestores e enfermeiros. Cad Saúde Pública [Internet]. 2015 Sep [cited 2017 Feb 22]; 31(9):1941-52. Available from: http:/ / dx.doi. org/10.1590/0102-311X00132114.

25. Sa LD, Oliveira AAV, Gomes ALC, Nogueira JA, Villa TCS, Collet N. Caring for tuberculosis patients in the Family Health Strategy: the nurses' perceptions. Rev Esc Enferm USP [Internet]. 2012 Apr [cited 2017 Mar 14]; 46(2):356-63. Available from: http:/ / www. scielo.br $/$ scielo.php?script $=$ sci_arttext\&pid $=$ S008062342012000200013\&lng=en\&nrm=iso\&tlng=en.

26. Härter J, Andrade RLP, Villa TCS, Arcêncio RA, RussoGonçalves E, Cardozo-Gonzales RI. Tuberculosis in primary health care: identifying priority cases in a municipality in southern Brazil. Acta Scientiarum - Health Sciences [Internet]. 2015 Jul/Dec [cited 2017 Mar 14]; 37(2):167-74. Available from: http:// periodicos.uem.br/ojs/index.php/ActaSciHealthSci/ article/view/25232/pdf_86.
27. Bulgarelli AF, Villa TCS, Pinto IC. Social organization and control of tuberculosis: the experience of a Brazilian town. Rev Latino-Am Enfermagem [Internet]. 2013 Sep/Oct [cited 2017 Mar 14]; 21(5):1039-48, Available from: http:/ / www.scielo.br/scielo.php?script=sci_ar ttext\&pid=S0104-11692013000501039.

28. Scatolin BE, Pinto ESG, Arcêncio RA, Andrade RLP, Wysocki AD, Ponce MAS, et al. Active case finding: community health workers' activity related to tuberculosis control in a large city, Brazil. Texto Contexto Enferm [Internet]. 2014 Apr/Jun [cited 2017 Mar 14]; 23(2):261-9. Available from: http:// www.scielo.br/scielo.php?script=sci_arttext\&pid $=$ S0104-07072014000200261

29. Marquieviz J, Alves IS, Neves EB, Ulbricht L. A Estratégia de Saúde da Família no controle da tuberculose em Curitiba (PR). Ciênc Saúde Coletiva [Internet]. 2013 Jan [cited 2017 Feb 22]; 18(1):265-71. Available from: http://dx.doi.org/10.1590/S1413-81232013000100027.

30. Bartholomay P, Pelissari DM, Araujo WN, Yadon ZE, Heldal E. Quality of tuberculosis care at different levels of health care in Brazil in 2013. Rev Panam Salud Publica. 2016 Jan [cited 2017 Mar 14]; 39(1):3-11.

31. Souza KMJ, Figueiredo DA, Villa TCS, SÁ LD, Nogueira JA, Palha PF. Tuberculosis treatment drop out and relations of bonding to the family health team. Rev Esc Enferm USP [Internet]. 2010 Dec [cited 2010 Mar 14]; 44(4):904-10. Available from: http://www. scielo.br/scielo.php?script=sci_arttext\&pid=S008062342010000400007\&lng=en\&nrm=iso\&tlng=en

32. Marcolino ABL, Nogueira JA, Ruffino-Netto A, Moraes RM, Sá, LD, Villa TCS, et al. Avaliação do acesso às ações de controle da tuberculose no contexto das equipes de saúde da família de Bayeux - PB. Rev Bras Epidemiol [Internet]. 2009 Jun [cited 2017 Mar 14]; 12(2):144-57. Available from: http://www. scielo.br/scielo.php?script=sci_arttext\&pid=S1415790X2009000200005

33. Figueiredo TMRM, Villa TCS, Scatena LM, Cardozo Gonzales RI, Ruffino Netto A, Nogueira JA, et al. Performance of primary healthcare services in tuberculosis control. Rev Saúde Pública [Internet]. 2009 [cited 2017 Mar 14]; 43(5):825-31. Available from: http://www. scielo.br/scielo.php?script=sci_arttext\&pid=S0034$89102009000500011 \& \operatorname{lng}=$ en\&nrm=iso\&tlng=en.

34. Trigueiro JVS, Nogueira JA, Sa LD, Palha PF, Villa TCS, Trigueiro DRSG. Tuberculosis control: decentralization, local planning and management specificities. Rev Latino-Am Enfermagem [Internet]. 2011 Nov/Dec [cited 2017Mar 14];19:1289-96. Available from: http://www.scielo.br/scielo.php?script=sci arttext\&pid=S0104-11692011000600003\&lng=en\&nr $\mathrm{m}=$ iso\&tlng $=$ en\&ORIGINALLANG $=$ en.

35. Marais, B, Lönnroth, K, Lawn, SD et al, Migliori GB, Mwaba P, Glaziou P, et al. Tuberculosis comorbidity with communicable and non-communicable diseases: integrating health services and control efforts. Lancet Infect Dis [Internet]. 2013 Apr [cited 2017 
Mar 14]; 13(4):285-7. Available from: http:/ / dx.doi. org/10.1016/S1473-3099(13)70015-X.

36. Curto M, Scatena LM, Andrade RLP, Palha PF, Assis EG, Scatolin BE, et al. Tuberculosis control: patient perception regarding orientation for the community and community participation. Rev LatinoAm Enfermagem [Internet]. 2010 Sep/Oct [cited 2017 Mar 14]; 18(5):983-9. Available from: http:// www.scielo.br/scielo.php?script=sci_arttext\&pid =S0104-11692010000500020

37. Oliveira SAC, Ruffino-Netto A, Villa TCS, Vendramini SHF, Andrade RLP, Scatena LM. Health services in tuberculosis control: family focus and community orientation. Rev Latino-Am Enfermagem [Internet]. 2009 May/Jun [cited 2017Mar 14];17(3):361-7. Available from: http:/ / www.scielo.br/scielo.php?script=sci_ar ttext\&pid=S0104-11692009000300013

38. Magnabosco GT, Lopes LM, Andrade RLP, Brunello MEF, Monroe AA, Villa TCS. Tuberculosis control in people living with HIV/AIDS. Rev LatinoAm Enfermagem [Internet]. 2016 Sep [cited 2017 Feb 22]; 24:e2798. Available from: http://dx.doi. org/10.1590/1518-8345.1187.2798. 